\title{
SU(2,1) Dynamics of Multiple Giant Dipole Resonance Coulomb Excitation
}

\author{
M.S. Hussein, A.F.R. de Toledo Piza, O.K. Vorov \\ Instituto de Fisica, Universidade de Sao Paulo \\ Caixa Postal 66318, 05315-970, \\ Sao Paulo, SP, Brasil
}

(13 August 1999)

\begin{abstract}
We construct a three-dimensional analytically soluble model of the nonlinear effects in Coulomb excitation of multiphonon Giant Dipole Resonances (GDR) based on the $\mathrm{SU}(2,1)$ algebra. The full 3-dimensional model predicts further enhancement of the Double GDR (DGDR) cross sections at high bombarding energies. Enhancement factors for DGDR measured in thirteen different processes with various projectiles and targets at different bombarding energies are well reproduced with the same value of the nonlinearity parameter with the exception of the anomalous case of ${ }^{136} \mathrm{Xe}$ which requires a larger value.
\end{abstract}


One of the most interesting applications of Coulomb Excitation in heavy ion collisions [1] is investigation of multi phonon nuclear Giant Resonances (GR) [2]. Possibility to excite multiple GR involves Bose statistics of collective excitations and constitutes the "family property" of vibrational collective motion in both finite (nuclei, clusters) and infinite quantum systems. Within this concept, making no distinction between infinite and finite systems, the excitation process can be modeled via a multidimensional quantum oscillator coupled linearly to the external time-dependent field, providing excellent agreement with the single-GR experimental data [2].

Validity of this completely linear theory has been questioned by nearly all the experimental data wherever multi-phonon GR has been observed [2]: the double Giant Dipole Resonance (GDR) excitation cross sections [8] are found about $1.3-2$ bigger than follows from theory. This shortcoming of the linear theory, known as "enhancement factor problem", has been addressed widely in current literature within a number of approaches: higher order perturbation theory [9], anharmonic effects [7], [10], concept of hot phonons [11 13]. Clearly, nonlinear effects, that in principle can not be neglected in finite Fermi systems [14], are not easily dealt with either at a microscopical or even at a phenomenological level [7].

It is therefore appealing to construct a natural, soluble "minimal extension" of the harmonic model of Coulomb excitations, in which deviations from the linear scheme are reasonably described via a few parameters. Without dealing in depth with the microscopic theory, we present here such a "minimal extension". This single-parameter nonlinear model can be solved exactly using algebraic properties of boson operator combinations forming algebra $\mathrm{SU}(2,1)$. The model allows us to correlate all the experimental data for the "enhancement factors" in various nuclei and various bombarding energies, using a single value of the universal nonlinear parameter.

Within the semiclassical approach [15] to Coulomb excitation, the projectile motion is approximated by a constant velocity $v$ on a straight line classical trajectory with impact parameter $b$ and internal excitation is treated quantum mechanically. The intrinsic state $|\Psi(t)\rangle$ of the system undergoing excitation obeys the time-dependent Schrödinger equation, 


$$
i \partial / \partial t|\Psi(t)\rangle=\left[H_{0}+V(t)\right]|\Psi(t)\rangle, \quad|\Psi(t=-\infty)\rangle=|0\rangle
$$

where $H_{0}$ is the intrinsic Hamiltonian and $V(t)=v_{1}(t)\left[\mathcal{D}_{-1}^{\dagger}-\mathcal{D}_{+1}^{\dagger}\right]+v_{0}(t) \mathcal{D}_{0}^{\dagger}+$ h.c. is the channel-coupling interaction with $\mathcal{D}^{\dagger}$ and $\mathcal{D}$ the dipole operators acting in the space of the multi-GDR states created by the boson operators $d_{m}^{+}$, with the angular momentum projection $m(\hbar=c=1)$. The functions $v_{1(0)}(t)$ describe the interaction with the electromagnetic field [2], [4] (see below). The excitation probability of an intrinsic state $|N\rangle$ with $\mathrm{N}$ phonons in a collision with impact parameter $b$ and the total cross section $\sigma_{N}$ are [2, 4, $\left., 5,15\right]$ :

$$
P_{N}(b)=|\langle N \mid \Psi(t=\infty)\rangle|^{2}, \quad \sigma_{N}=2 \pi \int^{\infty} b P_{N}(b)
$$

The internal nuclear Hamiltonian $H_{0}$ is nearly harmonic [2], [16], [17], so $H_{0}=\omega N=$ $\omega \sum_{m} d_{m}^{\dagger} d_{m}$. In principle, this does not exclude anharmonicities [18 in the transition operators $\mathcal{D}^{\dagger}, \mathcal{D}$ when expanded in terms of phonon operators

$$
\mathcal{D}_{m}^{\dagger}=d_{m}^{\dagger}+x \sum_{m_{1}} d_{m}^{\dagger} d_{m_{1}}^{\dagger} d_{m_{1}}+\sum_{m_{1}} x_{m_{1}} d_{m_{1}}^{\dagger} d_{m_{1}}^{\dagger} d_{m_{1}}^{\dagger}+x_{2} \sum_{m_{1} m_{2}} d_{m}^{\dagger} d_{m_{1}}^{\dagger} d_{m_{1}} d_{m_{2}}^{\dagger} d_{m_{2}}+\ldots
$$

These effective nonlinearities [14], [3] could result from perturbation theory treatment of anharmonicities in the phonon Hamiltonian, from coupling to other degrees of freedom, both collective (e.g., quadrupole GR) and noncollective [11] etc. The linear limit of the problem, $\mathcal{D}_{m}^{\dagger}=d_{m}^{\dagger}$, is exactly soluble giving the Poisson formula for the excitation probabilities

$$
P_{N}=e^{-\rho} \frac{\rho^{N}}{N !}, \quad \rho=\sum_{m=0, \pm 1}\left|\alpha_{m}^{h a r m}\right|^{2}=\sum_{m=0, \pm 1}\left|\int_{-\infty}^{\infty} v_{m}(t) e^{i \omega t} d t\right|^{2}
$$

where the amplitudes $\alpha_{m}^{\text {harm }}$ are given by the modified Bessel functions $K_{1}$ and $K_{0}$ [2].

In order to reduce the number of unknown parameters in Eq.(3) it is reasonable to restrict the higher-order nonlinear corrections $\propto x_{i}$. We save the first, dominating, nonlinear term in (3) with its coefficient $0 \leq x \ll 1$ and save only those terms in (3) which match terms appearing in the expansion of the square root, $\mathcal{D}_{m}^{\dagger} \rightarrow d_{m}^{\dagger}\left(1+2 x \sum_{m} d_{m}^{+} d_{m}\right)^{1 / 2}$.

The nonlinear effects are now controlled by the single parameter $x$. This may be justified, by noting that the leading term is saved, while the ansatz for the higher order terms, which 
should be small anyway, obeys the basic requirement that they get smaller as the order increases. Further, this parametrization leads to a soluble problem.

The group theoretical solution is based on the consideration of the eight operators

$$
\begin{array}{cc}
D^{+}=\left(d_{-1}^{+}-d_{+1}^{+}\right) \sqrt{k+N / 2}, \quad D_{0}^{+}=d_{0}^{+} \sqrt{2 k+N}, \quad J^{+}=\frac{1}{2^{1 / 2}}\left(d_{-1}^{+}-d_{+1}^{+}\right) d_{0}, \\
D^{0}=\frac{1}{4}\left[\left(d_{-1}^{+}-d_{+1}^{+}\right)\left(d_{-1}-d_{+1}\right)+2(2 k+N)\right], \quad D_{0}^{0}=\frac{1}{2}\left[2 k+N+d_{0}^{+} d_{0}\right] .
\end{array}
$$

and $D^{-}=\left(D^{+}\right)^{\dagger}, D_{0}^{-}=\left(D_{0}^{+}\right)^{\dagger}, J^{-}=\left(J^{+}\right)^{\dagger}$ with $k \equiv \frac{1}{4 x}$ the Casimir invariant. They form a closed $\mathrm{SU}(2,1)$ algebra, the non-compact analogue of $\mathrm{SU}(3)$. The pairwise commutators between (5) can be evaluated directly: Three of them are $\left[D^{-}, D^{+}\right]=2 D^{0},\left[D_{0}^{-}, D_{0}^{+}\right]=2 D_{0}^{0}$, $\left[J^{-}, J^{+}\right]=2\left(D_{0}^{0}-D^{0}\right)$. The other nonzero ones are $\left[D^{-}, D^{0}\right]=2\left[D^{-}, D_{0}^{0}\right]=\left[D_{0}^{-}, J^{-}\right]=D^{-}$; $\left[D_{0}^{-}, D_{0}^{0}\right]=2\left[D_{0}^{-}, D^{0}\right]=\left[D^{-}, J^{+}\right]=D_{0}^{-}$, and $\left[D^{-}, D_{0}^{+}\right]=2\left[J^{-}, D^{0}\right]=-2\left[J^{-}, D_{0}^{0}\right]=J^{-}$. The remaining nonzero commutators are given by Hermitean conjugates to the above. In the interaction representation, the evolution equation $i \frac{\partial}{\partial t}|\psi(t)\rangle=e^{i H_{0} t} V(t) e^{-i H_{0} t}|\psi(t)\rangle$ (1) with ([3) reads:

$$
i(\partial / \partial t)|\psi(t)\rangle=2 x^{1 / 2}\left[\tilde{v}_{1}(t) D^{\dagger}+\tilde{v}_{1}^{*}(t) D^{-}+\tilde{v}_{0}(t) D_{0}^{\dagger}+\tilde{v}_{0}^{*}(t) D_{0}^{-}\right]|\psi(t)\rangle .
$$

where $\tilde{v}_{1} \equiv v_{1} e^{i \omega t}$ and $\tilde{v}_{0} \equiv v_{0} e^{i \omega t} / \sqrt{2}$. Any product of exponentials involving the operators from the set (5) can be reduced to a simpler exponential (see, e.g., [19]), as due to closure of the pairwise commutators between (5), no new operator structures arise while re-arranging order of the operators in the time-ordered exponential (see, e.g., [20]) that solves (10),(6)

$$
|\psi(t)\rangle \equiv \operatorname{Texp}\left\{\int_{-\infty}^{t} e^{i H_{0} \tau} V(\tau) e^{-i H_{0} \tau} d \tau\right\}|0\rangle=e^{a D^{+}+b D_{0}^{+}} e^{f J^{+}} e^{c D^{0}+d D_{0}^{0}} e^{g J^{-}} e^{a^{\prime} D^{-}+b^{\prime} D_{0}^{-}}|0\rangle
$$

where the eight time-dependent c-numbers, the Latins $a-g$, must be chosen so $\psi(t)\rangle$ (7) obeys the Schrödinger equation (6). The ansatz (7) can be simplified using $D^{-}|0\rangle=D_{0}^{-}|0\rangle=$ $J^{ \pm}|0\rangle=0 ; D^{0}|0\rangle=D_{0}^{0}|0\rangle=k|0\rangle$, which follow from (5). The expression (7) then reduces to

$$
|\psi(t)\rangle=\left[1-4 x\left(|\alpha(t)|^{2}+\left.\beta(t)\right|^{2}\right)\right]^{\frac{1}{4 x}} e^{i \phi(t)} e^{\frac{\alpha(t)}{\sqrt{k}} D^{+}+\frac{\beta(t)}{\sqrt{k}} D_{0}^{+}}|0\rangle,
$$

where the first factor comes from unitarity, $\langle\psi(t) \mid \psi(t)\rangle=1$. The phase $\phi(t)$ is unimportant for the following. Substituting (8) into (6), we obtain, after some lengthy algebra using 
heavily the operator identity $e^{A} B e^{-A}=B+[A, B]+(1 / 2 !)[A,[A, B]]+\ldots$, the two nonlinear equations for the amplitudes $\alpha$ and $\beta$

$$
i \partial \alpha / \partial t-Q \alpha=\tilde{v}_{1}(t), \quad i \partial \beta / \partial t-Q \beta=\tilde{v}_{0}(t)
$$

with $Q \equiv 4 x\left[\tilde{v}_{0}^{*}(t) \beta+\tilde{v}_{1}^{*}(t) \alpha\right]$ and the initial condition $\alpha(-\infty)=\beta(-\infty)=0$. After projecting the asymptotic state (8) at $t=\infty$ onto the states with definite number of GDR phonons, we obtain the non-Poissonian expression for the excitation probabilities

$$
P_{N}=\frac{\Gamma\left(\frac{1}{2 x}+N\right)\left[4 x\left(|\bar{\alpha}|^{2}+|\bar{\beta}|^{2}\right)\right]^{N}}{N ! \Gamma\left(\frac{1}{2 x}\right)\left[1-4 x\left(|\bar{\alpha}|^{2}+\left.\bar{\beta}\right|^{2}\right)\right]^{-\frac{1}{2 x}}} .
$$

where $\bar{\alpha}$ and $\bar{\beta}$ are the asymptotic solutions of the system (9) at $t=\infty$. Their values can be easily tabulated by solving (9). If $x \rightarrow 0$, Eq.(10) is reduced to Poisson, while the solutions to (91) are reduced to the harmonic amplitudes (4), $|\alpha| \rightarrow\left|\alpha_{1}^{\text {harm }}\right|=\frac{2 F \xi^{2}}{\omega} K_{1}(\xi)$ and $|\beta| \rightarrow\left|\alpha_{0}^{\text {harm }}\right| / \sqrt{2}=\frac{2 F \xi^{2}}{\gamma \omega} K_{0}(\xi)$. Thus, the harmonic results are restored. At nonzero nonlinearity $x>0$, the multiple GDR excitation probabilities $P_{N}$ turn out to be enhanced as compared to their values $P_{N}^{\text {harm }}$ in the harmonic limit, Eq.(4). The cross sections $\sigma_{N}$ and their harmonic values, $\sigma_{N}^{\text {harm }}$ are given by integrating in Eq.(2) from the grazing value $1.2\left(A_{e x}^{1 / 3}+A_{s p}^{1 / 3}\right)$. The enhancement factors $r_{N}=\sigma_{N} / \sigma_{N}^{\text {harm }}$ can be studied and compared with the data. The functions $v_{m}(t)$, in the notations $\xi=\frac{\omega b}{v \gamma}, \tau=\frac{\omega t}{\xi}$ are [4. [4]

$$
v_{1}(t)=F f^{3}, \quad v_{0}(t)=F \sqrt{2} \gamma\left[-\frac{\partial f}{\partial \tau}-i \xi v^{2} f\right], \quad F=\frac{Z_{s p} e^{2} \gamma}{2 b^{2}}\left[\frac{N_{e x} Z_{e x}}{A_{e x}^{2 / 3} m_{N} \cdot 80 M e V}\right]^{\frac{1}{2}} .
$$

where $f=\left(1+\tau^{2}\right)^{-\frac{1}{2}}$ and $\gamma=\left(1-v^{2}\right)^{-\frac{1}{2}}$. In the strength $F, m_{N}$ and $e$ are the proton mass and charge, $Z, N$ and $A$ denote the nuclear charge, the neutron number and the mass numbers, the labels ex $(s p)$ refer to the excited (spectator) nucleus in the colliding pair.

Let us discuss the energy dependence of the two-phonon enhancement factor $r_{2}$. In Ref. [21], we have studied this behavior for the $\mathrm{Pb}+\mathrm{Pb}$ system at the bombarding energies in range $70-700 \mathrm{MeV} \cdot \mathrm{A}$, using truncated (two-dimensional) dynamics and neglecting the longitudinal response in the excitation process $\left(v_{0} \equiv 0\right)$. Within this "toy model" [21], the dynamics is 
described by the three operators $D, D^{+}$and $D^{0}$ which form the $\mathrm{SU}(1,1)$ subalgebra of the $\mathrm{SU}(2,1)$. The enhancement factor drops steadily as the bombarding energy grows. This is not the case at higher energies, when the transverse approximation breaks down and full solution based on the $\mathrm{SU}(2,1)$ algebra is required. This is illustrated in Fig.1a, where the enhancement factor $r_{2}$ is shown as a function of $\gamma$ for the case of $\mathrm{Pb}+\mathrm{Pb}$ collision in the energy range up to $4 \mathrm{Mev}$, as compared to the "transverse approximation".

The remarkable fact about the full 3-dimensional model is that the enhancement factor $r_{N}$ starts to grow again as the relativistic factor $\gamma$ passes the "extremal" point $(\sim 1.3-1.5$ for heavy colliding nuclei). Thus the 3 -dimensional $\mathrm{SU}(2,1)$ model predicts a new interesting qualitative effect which can be tested in experiments using higher bombarding energies. This behavior of $r_{N}$ is related to the $\gamma$-dependence of the "scaled" longitudinal function $\tilde{v}_{0}$ (Eq.11] [4.5]: $\tilde{v}_{0}(t)=v_{0}(t) e^{i \omega t}=F \sqrt{2}\left[-\gamma \frac{d}{d \tau}\left(f e^{i \xi \tau}\right)+i \frac{\xi}{\gamma} f e^{i \xi \tau}\right]$. The $\gamma$-dependence of the two terms here is very different. While the first term scales as $\gamma$, the absolute maximum of the second one scales as $\xi / \gamma=\gamma^{-2}$. The first term is proportional to the time derivative of the function $\frac{e^{i \omega t}}{\left(1+\tau^{2}\right)^{3 / 2}}$ which dies out at $t= \pm \infty$. Therefore, in the harmonic limit, when the longitudinal amplitude $\beta$ reduces to the simple integral over time of the function $\tilde{v}_{0}$, the first term simply vanishes. The harmonic solution is then given by the integral of the second term to give $\propto \frac{1}{\gamma} K_{0}(\xi)$ which dies out as $1 / \gamma$ as bombarding energy grows, and the longitudinal response becomes negligible at high bombarding energies as is well known [2 [4. This is not the case at nonzero nonlinearity $x \neq 0$ : the amplitude $\beta$ is given by the solution to the coupled nonlinear system (9), and the first term in $\tilde{v}_{0}$ not only does contribute but in fact becomes dominant. Unlike the second term, the first one grows with $\gamma$ and this determines the behavior of the enhancement factor.

We present below in Figs.1b, Fig.2 and Fig.3a exact numerical results for the cross sections calculated according to Eqs.(2), and (10) and using numerical solution of Eq.(9). Since the nonlinear parameter $x$ is an internal property of the nucleus in which the GDR is excited, it is reasonable to expect that it varies from one nuclear species to another. For the sake of comparison with the experimental data, it is expedient however to choose a single 
average value $x=0.29$ for all cases and use it to calculate the cross sections. As seen in Figs.1a,2 and Fig.3a, the nonlinear model can in this way (no fitting) reproduce rather well the experimental values [8] of $r_{2}$ for the twelve excitation processes of $\mathrm{Pb}$ (Fig.2a,b) and $\mathrm{Au}$ (Fig.2c), using different bombarding energies and targets/projectiles. An exceptional case is Xe, where considerably larger value of $x$ is required. To illustrate this, the optimal values of $x$ for each individual datum are shown in Fig.3b, together with the adopted average value.

In conclusion, we presented a simple soluble model to account for the nonlinear effects in the transition operators for the Coulomb excitation of multi-phonon GDR via relativistic heavy ion collisions. The solubility of the model is based on the group theoretical properties of the boson operators. It allows to construct the solution for the dynamics of the multiphonon excitation within the coupled-channel approach. The well known harmonic phonon model appears to be a limiting case of the present model when the nonlinearity goes to zero. The main advantages of the limiting harmonic case (unrestricted multiphonon basis, preservation of unitarity and possibility of analytical treatment) remain present in our nonlinear scheme. Therefore, the model can be viewed as a natural extension of the harmonic phonon model to include the nonlinear effects in a consistent way while keeping the model solvable. With proper modifications, it can be used in other (nuclear, molecule etc.) problems.

At low enough bombarding energies, the enhancement factor drops as the bombarding energy grows. This is consistent with the data and gives results similar to those recently obtained in a different context, with a theory based on the concept of fluctuations (damping) and the Brink-Axel mechanism [11], [12]. The interesting property of the full threedimensional results obtained here is that the enhancement factor starts growing again at high bombarding energies $(\sim 0.3-0.5 \mathrm{GeV} \cdot$ A for heavy colliding nuclei.) Besides being an interesting prediction to be tested in experiment, this behavior allows one to separate the effects of nonlinearity considered here and the effects proposed in works [1] 13].

The work has been supported by the FAPESP and by the CNPq. 


\section{REFERENCES}

[1] P.F.Bortignon, A.Braco and R.A.Broglia, Giant Resonances, Nuclear Structure at Finite Temperatures, (Harwood Acad., New York, 1998).

[2] H. Emling, Prog. Part. Nucl. Phys. 33, 729 (1994).

[3] T. Aumann, P.F. Bortignon, and H. Emling, Annu. Rev. Nucl. Part. Sci. 48, 351 (1998).

[4] C. A. Bertulani et al, Phys.Rev. C53, 334 (1996); C. A. Bertulani, nucl-ex/9803009.

[5] C. A. Bertulani and V. Yu. Ponomarev, Phys.Rep.,in press.

[6] G. Baur and C.A. Bertulani, Phys. Lett. B174, 23 (1986); P.G. Hansen, Nucl. Phys. A553, 89c (1993);J. Norbury and G. Baur, Phys. Rev. C48, 1915 (1993).

[7] P. Chomaz and N. Frascaria, Phys. Rep. 252, 275 (1995).

[8] R. Schmidt et al. Phys. Rev. Lett. 70, 1767 (1993); J.L.Ritman et al. Phys. Rev. Lett. 70, 533,2659 (1993); T.Aumann et al. Phys. Rev. C47, 1728 (1993); K.Boretzky et al., Phys. Lett. B384, 30 (1996); J.R. Beene, Nucl. Phys. A569, 163c (1994).

[9] C.A. Bertulani and V. Zelevinsky, Phys. Rev. Lett. 71, 967 (1993).

[10] P.F. Bortignon and C.H.Dasso, Phys. Rev. C56, 574 (1997).

[11] B.V.Carlson et al., Ann. Phys. (N.Y.), 1999, in press.

[12] B.V.Carlson, M. S. Hussein, and A. F. R. de Toledo Piza, Phys. Lett. B431, 249 (1998).

[13] B.V.Carlson et al., Phys. Rev. C60, 014604 (1999); B.V.Carlson et al., Phys.Rev. C59, 2689 (1999); B.V.Carlson and M.S.Hussein, Phys.Rev. C59, R2343 (1999).

[14] S.T. Belyaev and V.G. Zelevinsky, Nucl.Phys. 39, 582 (1962).

[15] K. Alder and A. Winther, Coulomb Excitation (Academic Press, New York, 1965).

[16] F.Catara, Ph. Chomaz and N. Van Giai, Phys. Lett. B233, 6 (1989). 
[17] G. F. Bertsch and H. Feldmeier, Phys.Rev. C56, 839 (1997).

[18] D.M. Brink, A.F.R. de Toledo Piza, and A.K.Kerman, Phys.Lett. 19, 413 (1965).

[19] J.N. Ginocchio and G. Wenes, in: Relations between Structure 66 Reactions in Nuclear Physics, Ed. D.H.Feng, M.Vallieres and B.H.Wildenthal, (World Scientific, Singapore, 1987) p.53; Y.Alhassid, F.Gürsey and F.Iachello, Phys. Rev. Lett. 50, 873 (1983).

[20] H. Kleinert, Fortschr. Phys. 26: 11, 565 (1978).

[21] M. S. Hussein, A. F. R. de Toledo Piza and O. K.Vorov, Phys. Rev. C59,R1242 (1999). 


\section{Figure Captions}

Fig.1. (a) The enhancement factor $r_{2}$ as a function of $\gamma$ for ${ }^{208} \mathrm{~Pb}+{ }^{208} \mathrm{~Pb}$ collision. The 3D results vs. the transverse approximation results ( $\mathrm{SU}(1,1)$ toy model, [21]) for $x=0.19$.

(b) Enhancement factor $r_{2}=\sigma_{2} / \sigma_{2}^{\text {harm }}$ for the DGDR excitation in ${ }^{208} \mathrm{~Pb}$ as a function of $\gamma$ (open symbols connected by a line). The data [8] (filled symbols): triangle up - Zn projectile, triangle right - U tagret, circles - $\mathrm{Pb}$ target and projectile. The value of the nonlinear parameter is kept fixed, $x=0.29$.

Fig.2. (a) The same as in Fig.1b, $x=0.29$. Square - Ar projectile, diamond - Kr projectile, triangle down - Ho, and circle - Sn. (b) The same for the DGDR in Au. Circle - Kr projectile, square - Au projectile, diamond - Bi projectile and triangle - Ne projectile. Open circles - theoretical values for the $\mathrm{Bi}$ projectile (the results for $\mathrm{Kr}$ and $\mathrm{Au}$ projectiles are the same at $\gamma \sim 2$ ).

Fig.3. (a) The same as in Fig.1b,Fig2, but for the DGDR in $X e, x=0.29$.

(b) The optimal values of the nonlinear parameter $x$ for the thirteen processes vs strength

parameter $F / \omega$, Eq. (11). The processes are numbered in the order they appear in Figs.1b, $2 \mathrm{a}, 2 \mathrm{~b}, 3 \mathrm{a}$ (from left to right). 
Hussein Fig. 1

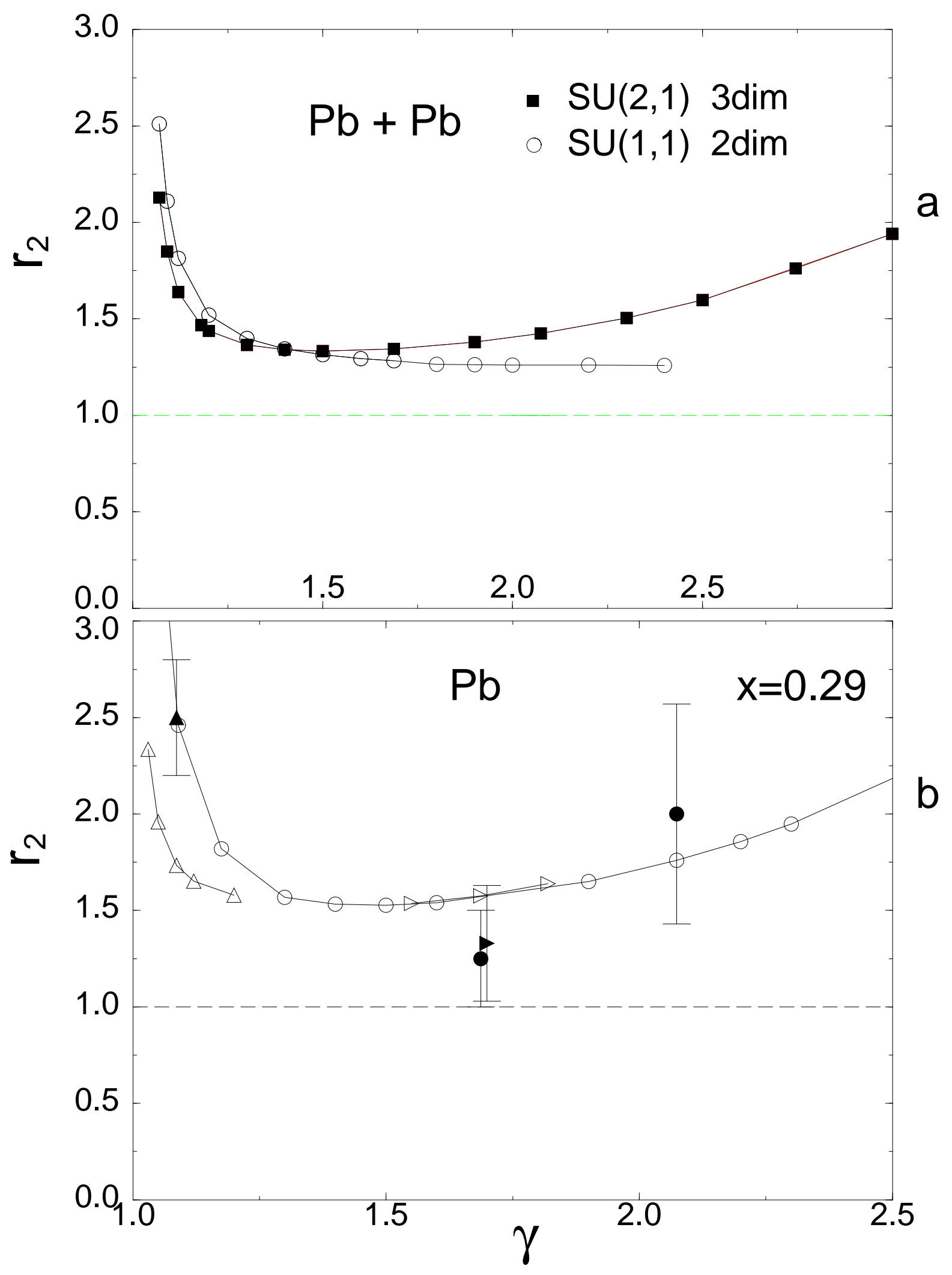


Hussein Fig. 2

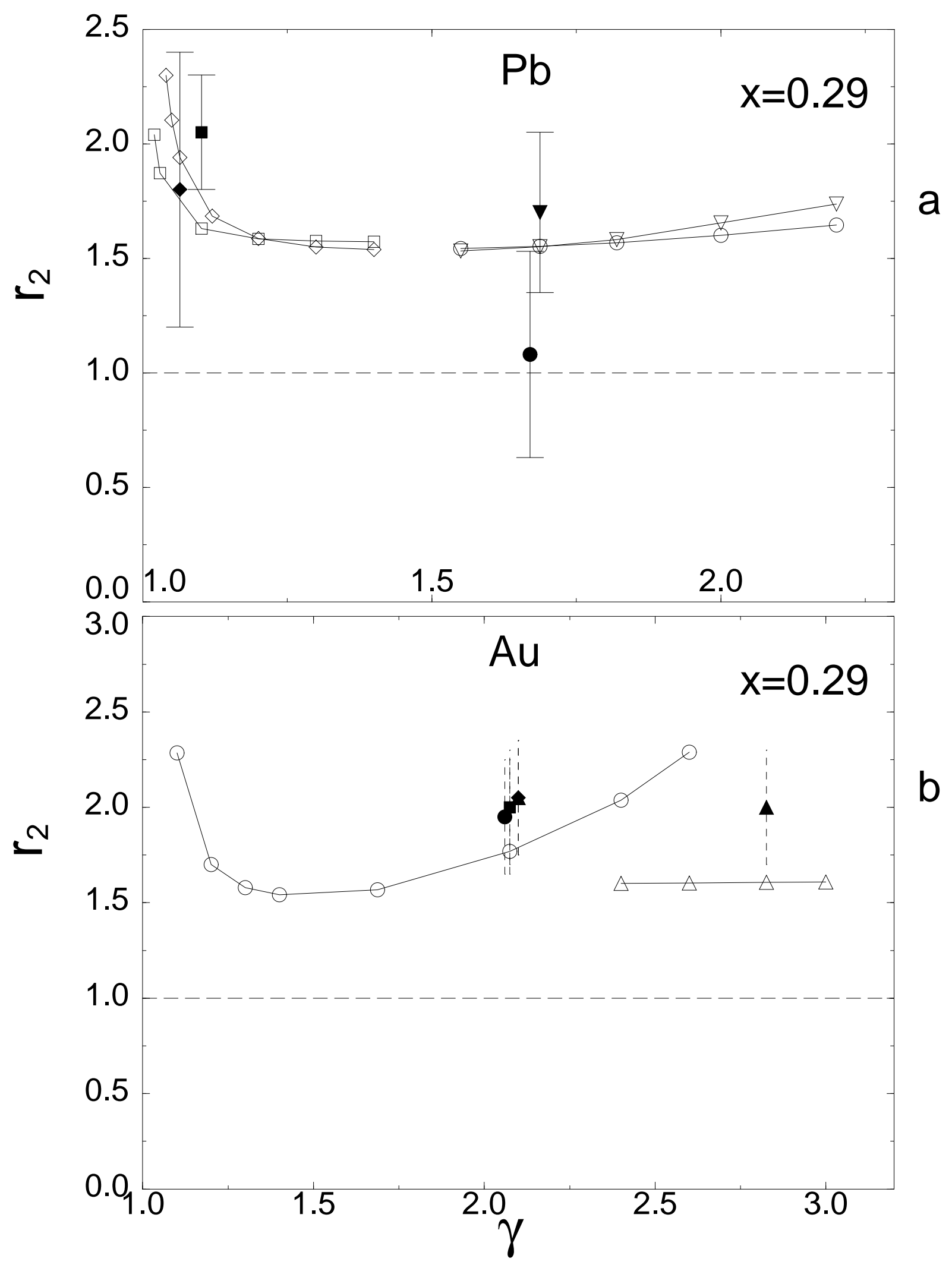


Hussein Fig. 3
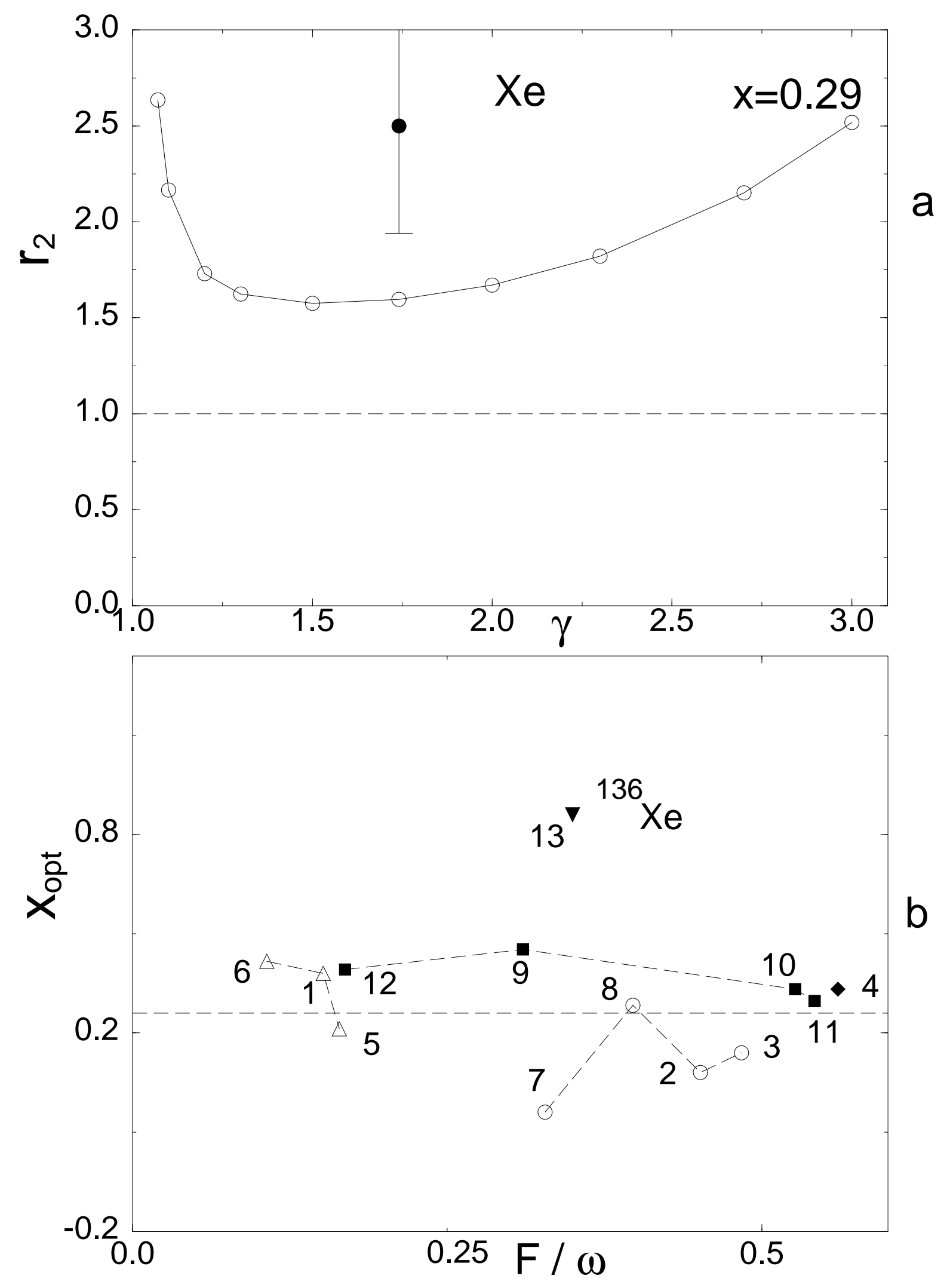